\title{
Preliminary Report of Pyrethroid Resistance in Anopheles vagus, an Important Malaria Vector in Bangladesh
}

\author{
Mohammad Shafiul Alam, ${ }^{1 \star}$ Hasan Mohammad Al-Amin, ${ }^{1,2}$ Wasif A. Khan, ${ }^{1}$ Rashidul Haque, ${ }^{1}$ Bernard L. Nahlen, ${ }^{3}$ and Neil F. Lobo ${ }^{3}$ \\ ${ }^{1}$ International Centre for Diarrhoeal Disease Research Bangladesh (icddr,b), Dhaka, Bangladesh; ${ }^{2}$ QIMR Berghofer Medical Research Institute \\ (QIMR Berghofer), Brisbane, Queensland, Australia; ${ }^{3}$ Eck Institute for Global Health (EIGH), University of Notre Dame, Notre Dame, Indiana
}

\begin{abstract}
According to the WHO, unmanaged insecticide resistance may lead to increases in malaria-related mortality and morbidity. Bangladesh, having made significant progress in malaria control efforts, has recently seen an upswing in malaria cases-58\% of which occurred in Bandarban district. Toward identifying entomological drivers of increased malaria, an entomological survey including Anopheles susceptibility to the insecticides in use was conducted in Bandarban. Anopheles vagus, the primary vector of malaria, was found to be resistant to both permethrin and deltamethrin-with only $29 \%$ and $55 \%$ mortality at 30 minutes, respectively. Intervention strategies in this area-all based on pyrethroids, may need to be reevaluated toward closing this gap in protection and increasing intervention efficacy.
\end{abstract}

Anopheles vagus (Donitz), has a wide distribution across Asia including the Indian subcontinent, the Greater Mekong Sub-region, and China. ${ }^{1}$ Anopheles vagus has been described as one of the most prevalent and widespread of the 36 Anopheles species documented in Bangladesh. ${ }^{2-5}$ This species was first documented as a malaria vector during an outbreak in 1993 (Kishoreganj district, Bangladesh). ${ }^{6}$ Anopheles vagus is believed to be the primary vector in Bangladesh as it is continuously documented in large numbers with high rates of Plasmodium infections, with a concurrent lower number, or lack of other primary vectors. ${ }^{2-4}$ This species has diverse oviposition sites that overlap with human habitation, including artificial containers-unusual for Anopheles mosquitoes. ${ }^{7}$ Other bionomic characteristics include primary exophily along with endophily, with anthropophilic as well as zoophilic feeding behavior., ${ }^{6,8}$ Overall, An. vagus is an efficient vector of malaria in Bangladesh.

Both government and private agencies rely primarily on insecticides to control Anopheles vectors. The National Malaria Elimination Program (NMEP) in Bangladesh has distributed deltamethrin-impregnated long-lasting insecticidal nets (LLINs) since 2008, with deltamethrin-based indoor residual spraying (IRS) being implemented in hotspots during outbreaks. ${ }^{9}$ However, this approach may be threatened by the rise and spread of insecticide resistance. ${ }^{10}$

Resistance of $A n$. vagus to dichlorodiphenyltrichloroethane (DDT) in Bangladesh has been reported since the 1980s. ${ }^{11} \mathrm{~A}$ limited NMEP study (between 2010 and 2015) suggested resistance to deltamethrin in An. vagus in Bandarban and Rangamati districts. ${ }^{9}$ Other Southeast Asian countries have reported insecticide resistance in this species to several pyrethroids including deltamethrin, permethrin, alphacypermethrin, and lambda-cyhalothrin. ${ }^{11}$ Whereas resistance has been indicated with organophosphate insecticides including malathion, susceptibility was seen with fenitrothion. ${ }^{10}$ Studies in Bangladesh have not conclusively demonstrated pyrethroid resistance in this vector. Major shortcomings in the NMEP study include the use of field-caught adult mosquitoes of unknown age, the recommended numbers of specimens tested remaining

* Address correspondence to Mohammad Shafiul Alam, International Centre for Diarrhoeal Disease Research Bangladesh (icddr,b), 68 Shaheed Tajuddin Ahmed Sarani, Mohakhali, Dhaka 1212, Bangladesh. E-mail: shafiul@icddrb.org unknown, and the lack of susceptible control mosquitoes. Toward addressing this knowledge gap, we investigated insecticide resistance against two major pyrethroids-deltamethrin and permethrin, using An. vagus specimens from the highest malarious district in Bangladesh, that is, Bandarban $\left(22.25^{\circ} \mathrm{N}\right.$, $\left.92.19^{\circ} \mathrm{E}\right)$, using CDC bottle bioassays ${ }^{12}$ along with insecticidesusceptible colony controls. This study was approved by the Research Review Committee and Ethical Review Committee of International Centre for Diarrhoeal Disease Research, Bangladesh (icddr,b).

Larvae were collected from three different areas in Bandarban toward a district-wide representative Anopheles sample. Collections were performed following WHO protocols, ${ }^{13}$ using a dipper in rice fields - the documented preferred larval site for An. vagus. ${ }^{7}$ Larvae, fed on ground fish food and dog biscuits, were reared to adulthood in the field insectary (icddr,b laboratory, Bandarban District Hospital). Sugar-fed 3to 5-day-old adult Anopheles, irrespective of species, were bioassayed for insecticide resistance. Mosquitoes were morphologically identified ${ }^{14}$ after insecticide resistance tests, and data from $A n$. vagus specimens were analysed for resistance. Low numbers of other Anopheles species rendered analyses of insecticide for these species unfeasible.

Bioassays were conducted using marked pre-coated bottles (Wheaton $250 \mathrm{~mL}$, Wheaton ${ }^{\circledR}$, Millville, NJ). ${ }^{12}$ Recommended insecticide concentrations used were as follows: $21.5 \mu \mathrm{g} / \mathrm{bottle}$ for permethrin and $12.5 \mu \mathrm{g} / \mathrm{bottle}$ for deltamethrin. ${ }^{12,15}$

Mortality data was recorded at 0,15 , and 30 minutes of exposure to each insecticide. Mosquitoes unable to stand were considered dead. According to WHO recommendations, ${ }^{15}$ data from bioassay tests were interpreted as follows: $\geq 98 \%$ mortality, susceptible; $90-97 \%$ mortality, developing resistance; and < 90\% mortality, resistant. High levels of resistance to permethrin ( $29 \%$ mortality, $n=131$ ) and deltamethrin (55\% mortality, $n=138$ ) were observed for An. vagus collected from larval habitats across Bandarban district.

Pyrethroid-based insecticides have been extensively used in Bangladesh. Permethrin-impregnated nets-distributed in bed net campaigns across Bangladesh from 2008 to 2013, were replaced with deltamethrin-impregnated LLINs over the last 10 years (NMEP, personal communication). Deltamethrin is also used for IRS in hotspots of malaria transmission. In addition, permethrin is widely used by local government authorities for mosquito control (primarily Aedes and Culex) in 
urban areas across the country. High levels of resistance in $A n$. vagus to both pyrethroids in the most malarious district are consistant with the historic use of this insecticide class and has important implications for malaria elimination in Bangladesh.

Limitations of this study include the small sample size and the need for more extensive collections across other regions of Bangladesh with malaria. The sampling of larval sites from across Bandarban enabled a regional evaluation of resistance to pyrethroids in the district that contributed to more than half the country's malaria burden in 2018. The inclusion of other insecticide classes and other Anopheles species would also be beneficial to decision-making. However, this study focused on the only insecticides (pyrethroids) in use and the primary vector in the region.

Bandarban district has consistently contributed to most of the cases in Bangladesh-ranging from $40 \%$ of reported malaria in 2015 to a high of $60 \%$ and $58 \%$ in 2017 and 2018 , respectively (NMEP, personal communication). The documentation of the primary vector-An. vagus, demonstrating resistance to the insecticide being used across a region with $58 \%$ of the country's malaria-signals a reduction in intervention efficacy that may have contributed to the doubling of reported human malaria infections between 2018 and 2019 (NMEP 2020, unpublished data). Although this small sample size should usually be inadequate to guide national policy, implications of the loss of insecticide efficacy in this district alone are consequential to the malaria burden in the entire country-demonstrating the requirement for more data across malaria-endemic regions. The evaluation of a national strategy toward malaria elimination requires the documentation of gaps in protection and their relationship to disease epidemiology. Systematic surveillance for insecticide resistance is a required part of these processes. As evident from other countries, evaluation of LLINs with synergists, ${ }^{16}$ IRS with other insecticides such as pirimiphos-methyl ${ }^{17}$ or carbamate and organophosphate, ${ }^{18}$ and alternative intervention strategies should be conducted toward combating characterized gaps in protection-including insecticide resistance.

Received February 24, 2020. Accepted for publication April 6, 2020.

Published online May 11, 2020.

Acknowledgments: icddr,b acknowledges with gratitude the Keough School of Global Affairs, and the Eck Institute for Global Health, University of Notre Dame, Notre Dame, IN, for funds received. icddr, $b$ is also grateful to the government of Bangladesh, Canada, Sweden, and the United Kingdom for providing core/unrestricted support.

Financial support: This study was funded by the Keough School of Global Affairs, and the Eck Institute for Global Health, University of Notre Dame, Notre Dame, IN.

Authors' addresses: Mohammad Shafiul Alam, Wasif A. Khan, and Rashidul Haque, International Centre for Diarrhoeal Disease Research Bangladesh (icddr,b), Dhaka, Bangladesh, E-mails: shafiul@icddrb.org, wakhan@icddrb.org, and rhaque@icddrb.org. Hasan Mohammad AlAmin, QIMR Berghofer Medical Research Institute (QIMR Berghofer), Brisbane, Australia, E-mail: hasan.al-amin@qimrberghofer.edu.au. Bernard L. Nahlen and Neil F. Lobo, Department of Biological Sciences, Eck Institute for Global Health (EIGH), University of Notre Dame, Notre Dame, IN, E-mails: bnahlen@nd.edu and nlobo@nd.edu.

\section{REFERENCES}

1. Rueda LM, Pecor JE, Harrison BA, 2011. Updated distribution records for Anopheles vagus (Diptera: culicidae) in the Republic of Philippines, and considerations regarding its secondary vector roles in southeast Asia. Trop Biomed 28: 181-187.

2. Alam MS, Khan MG, Chaudhury N, Deloer S, Nazib F, Bangali AM, Haque R, 2010. Prevalence of anopheline species and their Plasmodium infection status in epidemic-prone border areas of Bangladesh. Malar J 9: 15.

3. Alam MS et al., 2012. Diversity of anopheline species and their Plasmodium infection status in rural Bandarban, Bangladesh. Parasit Vectors 5: 150.

4. Al-Amin HM, Elahi R, Mohon AN, Kafi MA, Chakma S, Lord JS, Khan WA, Haque R, Norris DE, Alam MS, 2015. Role of underappreciated vectors in malaria transmission in an endemic region of Bangladesh-India border. Parasit Vectors 8: 195.

5. Irish SR, Al-Amin HM, Alam MS, Harbach RE, 2016. A review of the mosquito species (Diptera: culicidae) of Bangladesh. Parasit Vectors 9: 559.

6. Maheswary NP, Majumdar S, Chowdhury AR, Faruque MS, Montanari RM, 1994. Incrimination of Anopheles vagus Donitz, 1902 as an epidemic malaria vector in Bangladesh. Indian $J$ Malariol 31: 35-38.

7. Alam MS, Al-Amin HM, Elahi R, Chakma S, Kafi MAH, Khan WA, Haque R, Sack DA, Sullivan DJ Jr., Norris DE, 2018. Abundance and dynamics of Anopheles (Diptera: culicidae) Larvae in a malaria endemic area of Bangladesh. $J$ Med Entomol 55: 382-391.

8. Nagpal BN, Sharma VP, 1994. Indian Anopheless. New Delhi, India: Oxford \& IBH Publishing Co. Pvt. Ltd.

9. NMEP, 2017. National Strategic Plan for Malaria Elimination 2017-2021. Dhaka, Bangladesh: Directorate General of Health Services.

10. WHO, 2018. Global Report on Insecticide Resistance in Malaria Vectors: 2010-2016. Geneva, Switzerland: World Health Organization.

11. Mittal PK, Wijeyaratne P, Pandey S, 2004. Status of Insecticide Resistance of Malaria, Kala-Azar and Japanese Encephalitis Vectors in Bangladesh, Bhutan, India and Nepal (BBIN). Washington, DC: U.S. Agency for International Development.

12. CDC, 2010. Guidelines for Evaluating Insecticide Resistance in Vectors Using the CDC Bottle Bioassay: Enters for Disease Control and Prevention. Atlanta, GA: Center for Disease Control and Prevention.

13. WHO, 2003. Malaria Entomology and Vector Control (Learner's Guide). Geneva, Switzerland: World Health Organization.

14. Rattanarithikul R, Harrison BA, Harbach RE, Panthusiri $P$, Coleman RE, Panthusiri P, 2006. Illustrated keys to the mosquitoes of Thailand. IV. Anopheles. Southeast Asian J Trop Med Public Health 37: 1-128.

15. WHO, 2016. Test Procedures for Insecticide Resistance Monitoring in Malaria Vector Mosquitoes. Geneva, Switzerland: World Health Organization.

16. Menze BD, Kouamo MF, Wondji MJ, Tchapga W, Tchoupo M, Kusimo MO, Mouhamadou CS, Riveron JM, Wondji CS, 2020. An experimental hut evaluation of PBO-based and pyrethroidonly nets against the malaria vector Anopheles funestus reveals a loss of bed nets efficacy associated with GSTe2 metabolic resistance. Genes (Basel) 11: 143.

17. Abong'o B et al., 2020. Impact of indoor residual spraying with pirimiphos-methyl (Actellic 300CS) on entomological indicators of transmission and malaria case burden in Migori County, western Kenya. Sci Rep 10: 4518.

18. Yewhalaw D et al., 2017. Determination of the residual efficacy of carbamate and organophosphate insecticides used for indoor residual spraying for malaria control in Ethiopia. Malar J 16: 471. 